\title{
Reorganisation of stroke care in prehospital emergency medical services: results through transformative learning
}

\author{
Lauri Antila ${ }^{1, \star} \odot$, Anne Jyrkiäinen ${ }^{2} \odot$, Lasse Hakala ${ }^{1,3}$, Seppo Uusinarkaus ${ }^{1,3}$, \\ Juhani Tavasti ${ }^{1}$, Ari Palomäki ${ }^{1,4} \odot$
}

\author{
${ }^{1}$ Department of Emergency Medicine, \\ Kanta-Häme Central Hospital, \\ Ahvenistontie 20, FI-13530 \\ Hämeenlinna, Finland \\ ${ }^{2}$ Faculty of Education and Culture, \\ Tampere University, FI-33014 University \\ of Tampere, Finland \\ ${ }^{3}$ Emergency Medical Services of \\ Kanta-Häme, Kutalantie 1 B, FI-13210 \\ Hämeenlinna, Finland \\ ${ }^{4}$ Faculty of Medicine and Health \\ Technology, Tampere University, \\ FI-33014 Tampere University, Finland

\section{*Correspondence} \\ Lauri.Antila@khshp.fi \\ (Lauri Antila)
}

\begin{abstract}
Background: Rapid recanalisation is important when treating ischaemic stroke patients. In Finland, the reorganisation of the prehospital emergency medical system and the establishment of emergency medicine as an independent speciality occurred some years ago. These reforms offered the opportunity to develop new prehospital and in-hospital pathways for stroke patients.

Methods: In this retrospective study, we examined the immediate impact of implementing a new operating model in prehospital stroke care. We introduced a modified "load-and-go" model using a transformative learning process. We observed the immediate effects of the reorganisation by comparing prehospital time intervals three months before and three months after the reorganisation.

Results: The new operating model was implemented using a transformative learning process. There was an immediate reduction of $35.1 \%$ from 21.4 to 13.9 minutes $(P<$ $0.001)$ in the median on-scene time and of $18.2 \%$ from 52.7 to 43.1 minutes $(P<0.05)$ in the median total time, i.e. the time interval between the alarm from the dispatch centre to patient hand-over to ED.

Conclusion: By using a transformative learning process in implementing a modified load-and-go operation model in the EMS, we could immediately reduce median on-scene time and median total time in the treatment of acute stroke patients.
\end{abstract}

\section{Keywords}

Acute ischaemic stroke; Emergency medicine; Thrombolysis; Reorganisation; Prehospital emergency medical system

\section{Background}

Acute ischaemic stroke (AIS) patients benefit from rapid treatment by either intravenous thrombolysis with recombinant tissue plasminogen activator (tPA) or mechanical thrombectomy, as each minute after the onset of symptoms results in a cumulative loss of brain tissue, thereby significantly affecting the patient's prognosis $[1,2]$. In Finland, the reorganisation of prehospital emergency medical care and the introduction of emergency medicine as an independent speciality in its own right occurred in 2013 [3, 4]. These reforms have enabled the development of new prehospital and in-patient pathways for stroke patients.

In the Kanta-Häme Central Hospital (KHCH), a secondary care hospital in Southern Finland, we have reorganised stroke patients' in-hospital treatment pathway with promising results. With intensive training and a structured operating model, emergency physicians can safely treat patients with acute stroke in the Emergency Department (ED) without unnecessary delays. Further, the median door-to-needle time has decreased from
54 to 20 minutes $[5,6]$. However, treatment delays are not only dependent on in-hospital activity but also include the prehospital time intervals. This includes delays relating to the lack of public awareness of stroke symptoms and the overall functioning of emergency medical services (EMS) [7].

The American sociologist, Professor Jack Mezirow has developed a theory of transformative learning [8] where transformative learning refers to learning that renews a person's fundamental perceptions and relationship to the surrounding reality. When facilitating transformative learning, educators need to help people to understand their own and others' assumptions and to become critical of them. In suitable circumstances, through transformative learning, a person moves towards a frame of reference that is broader than earlier. By widening the world view, it is possible to change one's working practices [8].

Critical reflection is one of the key components of transformative learning $[9,10]$. Transformative learning is suitable for nursing education as well as basic and advanced medical education [11-13]. We thought that transformative learning 
might be a potentially useful tool in the training of paramedics and emergency medical technicians (EMTs) when introducing a new operating model. We hypothesised that through critical reflection prehospital EMS professionals would become aware of the new changes to the EMS process, 'unlearn' their old working practices and appreciate what the changes would mean when translated into daily practice.

Our EMS is owned by the town of Hämeenlinna and its operating area is around $5,700 \mathrm{~km}^{2}$. The medical management of the operation is in the hands of emergency physicians working in the ED of the Kanta-Häme Central Hospital, the surrounding municipalities together with the national health insurance fund EMS. We have two advanced life support level paramedics in every ambulance.

Soon after the reorganisation of the in-hospital pathway for AIS, we reorganised our prehospital operating model, from an earlier "stay and play" principle towards a modified "load and go" principle for suspected stroke patients. In this retrospective study, we present this reorganisation including the immediate short-term results of this action. The local ethics committee approved the study.

\section{Methods}

This was a retrospective study in which immediate changes in time intervals were analysed. First, we present the reorganisation of our prehospital operating model for stroke patients in the EMS. Second, we report the rapid impact of the intervention on prehospital delays three months after the reorganisation.

\subsection{Reorganisation}

This reorganisation was carried out in three basic steps.

\subsubsection{Analysis and reform of the AIS operating model}

The operating model was reformed in order to optimise the patients' pathway from the AIS alarm at the Dispatch Centre to arrival at the ED of $\mathrm{KHCH}$. According to the old stroke operating model, EMS personnel were required to carry out a routine physical examination of the patient, focusing on vital and neurological signs. However, clear triggers for a rapid treatment decision in suspected AIS were lacking. The new operating model was based on immediate recognition of stroke symptoms and rapid decision-making on transportation to the ED (Table 1). We used Face Arm Speech Test (FAST) with findings of possible eye deviation to screen suspected AIS $[14,15]$. In prehospital EMS, the assessment of possible stroke must be sensitive so that all patients at risk are transported to the ED. Findings of eye deviation were added as a marker of possible stroke in order to improve sensitivity. In addition, examination and immediate management on scene were planned in order to concentrate solely on the most important immediate clinical issues relevant to the rapid and reliable diagnosis and treatment of the patient. All other interventions, including obtaining IV access, were recommended to be carried out safely during transportation. We have a reversible chair in all ambulances in order to do the procedures properly. The seat belts are always fastened when the ambulance is in motion. Paramedics informed the ED of the patient's arrival en route in order to avoid any additional delays. If the estimated arrival time at the hospital was less than 15 minutes, pre-notification had to be given on site in order to allow the ED personnel adequate preparation time.

\subsubsection{Information for personnel}

EMS personnel was taught the new operating model and its justification was discussed. The emergency physician, along with the senior paramedics, provided education. It was both theoretical and practical. However, we did not provide any additional simulation training in this regard. From an educational point of view, our aim was to encourage paramedics to research, investigate, critically reflect on and discuss the new operating model. The hypothesis was that this would serve to corroborate the recommended actions in the new operating model [9]. The operating model was presented to the EMS personnel in a total of 16 events at four different ambulance stations. The main educational element was discussion about the new operating model and education on how to recognize the main symptoms of AIS and to allow fast decision-making to transport the patient to the ED. The written operating model was then sent to the EMS personnel via email and processed through educational sessions in groups of two to ten staff led by the EMS supervisors.

\subsubsection{Implementation of the new operating model}

The most important factor in the implementation of the operating model was the use of a transformative learning process [10]. During this process, we challenged paramedics to change the culture of full physical examination of suspected stroke patients ("stay and play") to fast recognition of main symptoms, prompt decision-making and rapid transportation to hospital ("load and go"). Following the educational meetings and events, we also maintained awareness by three articles published in the personnel magazine. The key to a successful implementation was thought to be regular updating on the success of the project and a positive drive. We encouraged EMS personnel to actively consider why it is important to start immediate transportation. We wanted especially to improve staff understanding of the fact that there is very little to be done on scene that will positively influence a patient's prognosis.

Transformative theory emphasizes that through critical reflection, it is possible to transform pre-existing assumptions upon which interpretations, beliefs and habits of mind or point of view are based [10]. We wanted to avoid any implication that we were consciously underestimating professionals' skills due to the introduction of a simplified operating model. Instead, we aimed to create a culture of excellence [16] and the understanding that "less is more" in the high-quality stroke care on scene. In order to reinforce our message, we attached stickers to ambulances, cell phones and EMS notepads. We also reminded personnel about the FAST principle with posters on the walls of all the ambulance stations (Fig. 1). Transformative learning also occurs in individual and social contexts. This process was realised as described above, and by encouraging EMS personnel to discuss, question and reflect on 
TA B L E 1. Suspected stroke in emergency medical care. Key points of the new prehospital operating model Rapid and reliable recognition of possible stroke

- Abnormal finding in FAST

AND/OR

- Eye deviation

Only the most important tasks performed on scene

- BP, HR, Sats, GCS

- No complete physical examination

- Basic information: personal identification, contact details of a relative, functional status before stroke symptoms (documenting "last-known-well” time) and list of patient's medication(s)

- Most important: exact time of stroke symptoms onset (or last time patient was seen before symptoms)

Other interventions during transportation

- POC measurement of blood glucose

- IV line with saline infusion

- Monitoring and treatment of BP, HR or Sat

- Closer physical examination of the patient

- Second IV line without infusion when other tasks are done

Pre-notification to emergency department

Abbreviations: BP, blood pressure; FAST, Face Arm Speech Test; GCS, Glasgow Coma Scale; HR, heart rate; IV, intravenous; POC, point-of-care; Sats, blood oxygen saturation.

their experiences in our meetings.

\section{Best in the world}

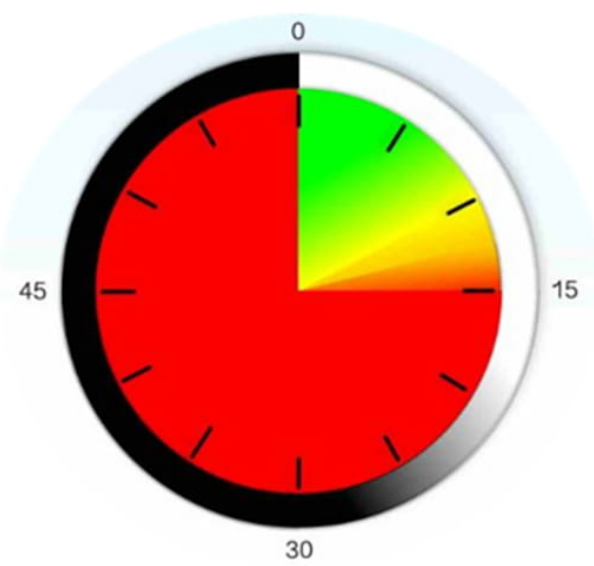

\section{Acute Ischaemic Stroke - Time Matters!}

Every minute approximately two million neurons die

Our goal: On-scene time less than 10 minutes

We do it with excellent and fluent cooperation

\section{We Have the Capability!}

FIGURE 1. Poster designed to encourage EMS personnel.

In practice, there was some overlap between the different phases of our three-step programme. According to transformative theory, the core of our reorganisation was to establish how EMS personnel, both individually and as a team, could expedite the prehospital pathway for stroke patients.

\subsection{Time intervals}

We compared prehospital time intervals during the threemonth period immediately following the reorganisation with those over the preceding three months. All patients transported by the emergency medical system AIS code to the ED of Kanta-Häme Central Hospital were included in the study.

The following time intervals were analysed: 1. Ambulance response time from alarm to arrival on scene, 2. On-scene time (OST, time spent on scene), 3. Transportation time (time between start of transportation and patient hand-over from EMS to ED), and 4. Total time (alarm from dispatch centre to patient hand-over to ED). We took timings from the SQL database of the EMS system and then double-checked from the corresponding handwritten EMS documents. Our specific aim was to analyse whether the reorganisation affected the onscene time among patients with suspected AIS.

\subsection{Statistical methods}

Statistical analyses were performed using IBM $^{\circledR}$ SPSS ${ }^{\circledR}$ Statistics for Windows, version 23 (IBM Corp., Armonk, N.Y., USA; copyright 2015). Data are presented as median (minimummaximum) unless otherwise stated. Differences in continuous variables were assessed using the nonparametric MannWhitney U-test for independent samples. A probability value $<0.05$ was considered statistically significant. 


\section{Results}

Our EMS completed the first two steps (analysis and reform of AIS operating model and its dissemination to personnel) during the last weeks before the official reorganisation. The third step (implementation of the new operating model) was commenced at the onset of the reorganisation, but the process is continuous in nature.

\subsection{Reorganised EMS operating model}

EMS reorganised the new stroke operating model within in the time period envisaged. The paramedics put obligatory steps for managing all suspected AIS patients into practice as follows: the use of a modified FAST, the assessment of vital signs together with point-of-care measurement of capillary blood glucose, documenting "last-known-well"-time and performing hospital pre-notification. During handover, paramedics began their reporting to ED personnel using the structured ISBAR (Introduction, Situation, Background, Assessment and Recommendations) tool [17]. Because the long-term goal of an on-scene time of less than 10 minutes was set for all EMS personnel in potential stroke patients (Fig. 1), various tasks were commenced in a precise order. For example, ongoing medication had to be established, but only if this was feasible in less than five minutes. Otherwise, we had to rely on patient records at the hospital.

\subsection{Time delays}

The total number of suspected AIS patients transported to the $\mathrm{ED}$ of $\mathrm{KHCH}$ was 52 before and 57 following the reorganisation of services. There were a total of 60 men and 49 women in the whole study group, 26 and 34 men and 26 and 23 women before and after the reorganisation respectively (NS). Their median age was 71.2 (SD 15.0) years. There was no difference in patients' ages between the two study periods (Table 2). The results of the time intervals are presented in Fig. 2 and Table 2. During the first three months of the reorganisation, OST decreased by $35.1 \%$ or 7.5 minutes from 21.4 to 13.9 minutes $(P<0.001)$ and total time by $18.2 \%$ or 9.6 minutes from 52.7 to 43.1 minutes $(P<0.05)$ compared to the preceding three-month period. There were no differences in any of the prehospital time intervals between men and women (data not shown). When the alarm from the Dispatch Centre included the code "Stroke", median OST over the whole study period (i.e. before and after the reorganisation) was $31.8 \%$ shorter than without this code, $14.5 \mathrm{~min} v s .21 .3 \mathrm{~min}$, respectively $(P<0.001)$.

\section{Discussion}

In this retrospective study, we presented an effectively reorganised prehospital EMS operating model for acute stroke patients. Our main finding was that by using a transformative learning process the new operating model resulted in an immediate reduction in on-scene time.

To the best of our knowledge, this is the first study of its kind to report the implementation of transformative learning theory as a framework for the reorganisation of a prehospital

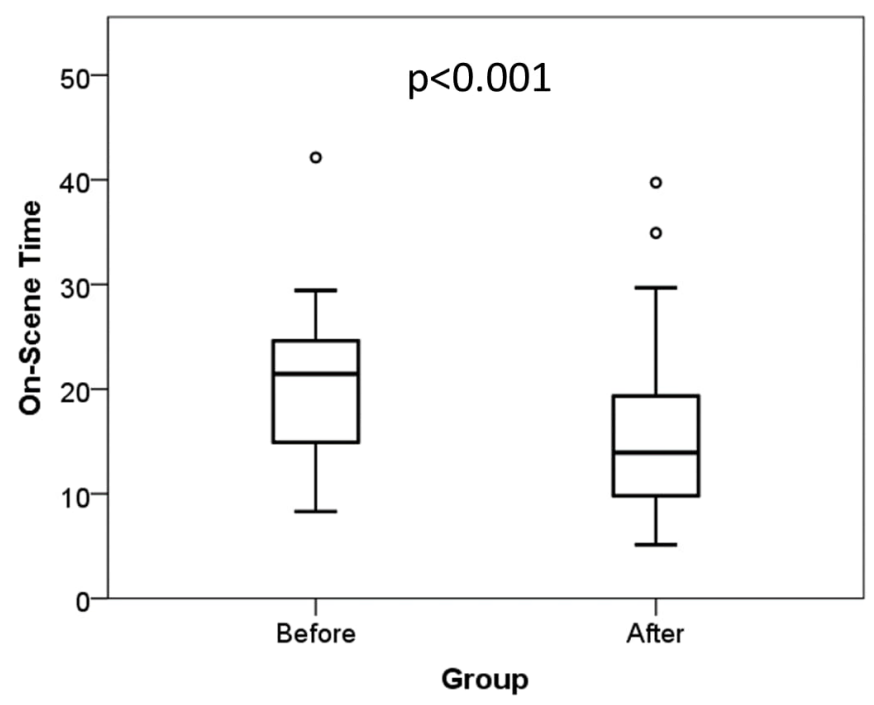

F I G U R E 2. On-Scene Time comparison before and after the reorganisation $(P<0.001)$.

operating model for stroke patients in EMS. We carried out a search in PubMed (Publication dates: $1^{\text {st }}$ January 2000 to $10^{\text {th }}$ December 2020; search terms "transformative learning" AND "acute" AND "stroke" AND ("emergency medical services" OR "EMS" OR "prehospital") that did not yield any publications. Critical reflection has been shown to be a significant feature. During this process, we supported paramedics to critically reflect, think and discuss the assumptions on which their beliefs were founded in the prehospital operating model for the treatment of stroke patients. Reflection enabled paramedics to change their actions in order to positively influence patients' prognoses and to challenge their own beliefs and possible errors in treating patients with AIS.

Our EMS system for detecting AIS relies on a modified FAST having only three options: Positive (leading to immediate highest priority transportation to hospital, pre-notification with no expert consultation), Unreliable (expert consultation needed to end up with stroke code) or Negative. Good agreement between paramedics and physicians has been reported earlier in the recognition of neurological deficits by using FAST (14). This concurs with the findings of Yanagida et al. that severity of symptoms had an effect on prehospital delays; thus, presence of low level of consciousness, aphasia, headache or nausea ended in shorter prehospital delays than did absence of these factors [18].

The use of EMS reduces prehospital delays [19]. Other interventions introduced by EMS have an effect not only on prehospital time intervals, but also on in-hospital measures [20-22]. An OST of less than 15 minutes, transportation at highest priority, documentation of "last-known-well"-time, and the use of a prehospital stroke assessment tool are considered to be indicators of quality for EMS [22]. These findings are in accordance with the principles of our reorganisation. Our reduction of on-scene time also compares favourably with published data from different operational environments in Europe [23-27].

The total time of our EMS was already less than one hour and it was immediately reduced by $18.2 \%$ following the reorgani- 
TA B L E 2. Prehospital time intervals of suspected stroke patients

\begin{tabular}{lccc} 
& Before & After & $P$-value \\
Number of patients & 52 & 57 & \\
\hline Age, years & $70.6(25-97)$ & $71.7(32-99)$ & NS \\
\hline Ambulance response time & $a$ \\
OST $^{b}$, minutes & $7.6(2.5-32.0)$ & $6.8(1.5-31.0)$ & 0.70 \\
\hline Transportation time $^{c}$, minutes & $21.4(8.3-42.1)$ & $13.9(5.1-39.7)$ & $<0.001$ \\
\hline Total time, minutes & $24.5(2.9-85.8)$ & $21.8(1.6-46.9)$ & 0.58 \\
\hline
\end{tabular}

All times are presented as median times (minimum - maximum times in brackets). NS, not significant.

a) Time from alarm to arrival on scene; b) On-Scene time; c) Transportation Time, from Transportation start to emergency department.

sation. In a study by Sundström et al. the median delay from AIS emergency call to CT scan was 3 hours and 52 minutes in 2015 [28]. The study also found that in western Sweden highest mission priority was only given to $54 \%$ of cases. In a South Korean EMS in 2016 it was reported that $30.8 \%$ of AIS patients transferred by EMS reach the emergency department within 2 hours. Transportation by EMS, straight to a unit capable providing thrombolytic therapy was the fastest way to achieve a high standard of care [29]. It is worth pointing out that the total time does not only depend on the quality of prehospital EMS. The location of the hospital and geographical features of its surroundings (e.g., narrow roads, hills, or large lakes) and distances (e.g., from the scene to the hospital) are important variables. Therefore, the results obtained in different countries or regions cannot be directly compared. In our case, the province is compact in size and the access routes to the hospital are good.

In a large study in the USA, hospital pre-notification occurred in $67 \%$ of AIS cases and an association was found between pre-notification and timelier in-hospital treatment [20]. According to two recent studies, hospital pre-notification was performed in $52 \%$ to $77 \%$ of patients $[30,31]$. We did not formally evaluate the percentage of pre-notification, but according to reports from ED personnel, notification was given in at least $90 \%$ of suspected AIS cases (Iiro Heikkilä, personal communication). It has been found that dispatching fire engine crews as first responders in order to help EMS crew does not reduce on-scene time [32]. According to our operating model, the crew taking care of patients on the stroke prehospital pathway consists solely of paramedics.

One strength of this study is that it was carried out in a limited time frame. Seasonal variation in the weather, for example frost and snow in winter, did not affect our results and bias the immediate effects of the reorganisation. Furthermore, when planning the new prehospital operating model, we relied on our previous experience in the reorganisation of the emergency department and its operations [3, 33, 34], including in-hospital door-to-needle delay of stroke patients [5, 6]. In our reorganisation, we chose to implement modified FAST method to trigger a stroke code in the EMS system because it has a high positive predictive value in detecting AIS patients, especially when implemented on scene [14, 35]. The detecting algorithm should be sensitive rather than specific for stroke [36].
In this study, the background to reorganising prehospital EMS was based on a transformative learning process. It clearly highlights the importance of taking into account the EMS personnel's experiences, expectations and reflection during the process. Hence, we obtained a more detailed understanding of the learning processes of paramedics during the reorganisation as we evaluated their experiences by interview and enquiry. By eliciting personnel's significant learning experiences, it was possible to find the best way to support paramedics and to increase the effectiveness of our EMS operating model for stroke patients. Although transformative learning has not traditionally been common in medicine, it has recently attracted attention in medical education [37]. We want to emphasise that we did not compare transformative learning to any other learning method in this study.

This was a retrospective study with a before and after setting. We did not implement a prospective study to compare, for example, two different hospital areas with each other. This could be cited as a limitation of this study. Pieri et al. have recently reminded us about the Hawthorne effect, also called the observer effect, which means that subjects who know their actions are being studied improve or modify aspects of their behaviour in response to the awareness of being studied [38]. Furthermore, there is a saying in management and quantitative research: "What you measure is what you get". All of our paramedics were aware of our goal to shorten prehospital delays and of the aim to measure such delays. Therefore, it was not possible to differentiate the effect of the transformative learning process from the motivation that had arisen from the goalsetting itself. Nor was it possible to measure learning through reflection. However, it gave us a more detailed understanding of the EMS personnel's experiences and feelings whilst learning and implementing the new operating model during the reorganisation process. On the other hand, goal setting could be seen as a part of the learning process as a whole. Although we achieved an immediate reduction of prehospital time intervals, the follow-up period of this study was too short to confirm that these significant changes were permanent. We are therefore continuing the process reported here, not only as a part of our clinical work but also in delineating future research. 


\section{Conclusions}

In this study, we used a transformative learning process for EMS personnel when implementing a modified load and go operation model in the treatment of acute stroke patients. By so doing we could immediately reduce the median on-scene time from 21.4 to 13.9 minutes and the median total time (alarm from dispatch centre to patient hand-over to ED) from 52.7 to 43.1 minutes. Although we need longer follow-up to evaluate the stability of our findings, this study shows that rapid change in operating model is possible in prehospital EMS.

\section{ABBREVIATIONS}

AIS, Acute ischaemic stroke; BP, Blood pressure; ED, Emergency Department; EMS, Emergency medical system; EMT, Emergency medical technician; FAST, Face Arm Speech Test; GCS, Glasgow Coma Scale; HR, Heart rate; IV, Intravenous; KHCH, Kanta-Häme Central Hospital; NS, Not significant; OST, On-Scene Time; POC, Point-of-care; Sats, Blood oxygen saturation; tPA, Tissue plasminogen activator.

\section{AUTHOR CONTRIBUTIONS}

AP designed the study; JT, LH and SU implemented the learning process outlined by AJ. LH and SU organised the data collection; AP carried out statistical analyses; LA, LH, AJ, JT and AP drafted the manuscript. All authors critically revised the manuscript, and, finally, read and approved it.

\section{ETHICS APPROVAL AND CONSENT TO PARTICIPATE}

The ethics committee of Tampere university hospital approved the study (R19021).

\section{ACKNOWLEDGMENT}

The authors gratefully acknowledge the professional technical aid of Anni Innamaa, Iiro Heikkilä, and Virginia Mattila as well as the co-operation of the EMS staff working in the Emergency Medical Services of Kanta-Häme.

\section{FUNDING}

The study was funded by the Ministry of Health and Social Welfare in Finland via the Medical Research Funds of KantaHäme Central Hospital and Tampere University Hospital.

\section{CONFLICT OF INTEREST}

The authors have not declared any competing interests.

\section{AVAILABILITY OF DATA AND MATERIALS}

The datasets used and analysed during the present study are available from the corresponding author on reasonable request.

\section{AUTHORS' INFORMATION}

LA works as a trainee of EM in the $\mathrm{KHCH}$, Hämeenlinna. $\mathrm{AJ}$ is a university lecturer and acts as the vice dean in the Tampere University, Faculty of Education and Culture. LH works as the manager and SU as the supervisor in the EMS Kanta-Häme, Finland. JT has been working as the deputy medical director in $\mathrm{KHCH}$, Hämeenlinna. AP is Professor of emergency medicine at Tampere University, Faculty of Medicine and Health Technology, and the medical director of the ED and the Division of Medical Specialities in the $\mathrm{KHCH}$, Hämeenlinna, Finland.

\section{REFERENCES}

[1] Meretoja A, Strbian D, Mustanoja S, Tatlisumak T, Lindsberg PJ, Kaste M. Reducing in-hospital delay to 20 minutes in stroke thrombolysis. Neurology. 2012; 79: 306-313.

[2] Meretoja A, Keshtkaran M, Saver JL, Tatlisumak T, Parsons MW, Kaste M, et al. Stroke thrombolysis: save a minute, save a day. Stroke. 2014; 45: 1053-1058.

[3] Naskali J, Palomäki A, Harjola VP, Hällberg V, Innamaa T, Rautava VP. Emergency medicine in Finland: first year experiences of specialist training. Journal of Academic Emergency Medicine. 2014; 13: 26-29.

[4] Baldursson J, Björnsson HM, Palomäki A. Emergency medicine for 25 Years in Iceland - history of the specialty in a nutshell. Scandinavian Journal of Trauma, Resuscitation and Emergency Medicine. 2018; 26: 1.

[5] Heikkilä I, Kuusisto H, Stolberg A, Palomäki A. Stroke thrombolysis given by emergency physicians cuts in-hospital delays significantly immediately after implementing a new treatment protocol. Scandinavian Journal of Trauma, Resuscitation and Emergency Medicine. 2016; 24: 46.

[6] Heikkilä I, Kuusisto H, Holmberg M, Palomäki A. Fast operating model for treating acute ischemic stroke by emergency physicians. Annals of Emergency Medicine. 2019; 73: 105-112.

[7] Kim YS, Park SS, Bae HJ, Cho AH, Cho YJ, Han MK, et al. Stroke awareness decreases prehospital delay after acute ischemic stroke in Korea. BMC Neurology. 2011; 11: 2.

[8] Mezirow J. Transformative learning: theory to practice. New Directions for Adult and Continuing Education. 1997; 1997: 5-12.

[9] Dewey J. How we think. Boston: D.C. Heath \& Co. 1910.

[10] Mezirow J. Learning as transformation: critical perspectives on a theory in progress. The Jossey-Bass Higher and Adult Education Series. San Francisco: Jossey-Bass Publishers. 2000.

[11] Tsimane TA, Downing C. Transformative learning in nursing education: a concept analysis. International Journal of Nursing Sciences. 2019; 7: 91-98.

[12] Kerins J, Smith SE, Phillips EC, Clarke B, Hamilton AL, Tallentire VR. Exploring transformative learning when developing medical students' non-technical skills. Medical Education. 2020; 54: 264-274.

[13] Foshee CM, Mehdi A, Bierer SB, Traboulsi EI, Isaacson JH, Spencer A, et al. A professionalism curricular model to promote transformative learning among residents. Journal of Graduate Medical Education. 2017; 9: 351356.

[14] Nor AM, McAllister C, Louw SJ, Dyker AG, Davis M, Jenkinson D, et al. Agreement between ambulance paramedic- and physician-recorded neurological signs with Face Arm Speech Test (FAST) in acute stroke patients. Stroke. 2004; 35: 1355-1359.

[15] Wu Y, Tsai T, Huang C. Contraversive shift of conjugate eye deviation in hemispheric stroke indicates emerging mass effect. Journal of Clinical Neuroscience. 2011; 18: 1276-1278.

[16] Campbell AR. Building a culture of excellence from the ground up. Radiology Management. 2013; 35: 14-18.

[17] Dawson S, King L, Grantham H. Review article: Improving the hospital clinical handover between paramedics and emergency department staff in the deteriorating patient. Emergency Medicine Australasia. 2013; 25: 
393-405.

[18] Yanagida T, Fujimoto S, Inoue T, Suzuki S. Prehospital delay and strokerelated symptoms. Internal Medicine. 2015; 54: 171-177.

[19] Kim DH, Nah HW, Park HS, Choi JH, Kang MJ, Huh JT, et al. Impact of prehospital intervention on delay time to thrombolytic therapy in a stroke center with a systemized stroke code program. Journal of Stroke and Cerebrovascular Diseases. 2016; 25: 1665-1670.

${ }^{[20]}$ Lin CB, Peterson ED, Smith EE, Saver JL, Liang L, Xian Y, et al. Emergency medical service hospital prenotification is associated with improved evaluation and treatment of acute ischemic stroke. Circulation: Cardiovascular Quality and Outcomes. 2012; 5: 514-522.

[21] McKinney JS, Mylavarapu K, Lane J, Roberts V, Ohman-Strickland P, Merlin MA. Hospital prenotification of stroke patients by emergency medical services improves stroke time targets. Journal of Stroke and Cerebrovascular Diseases. 2013; 22: 113-118.

[22] Oostema JA, Nasiri M, Chassee T, Reeves MJ. The quality of prehospital ischemic stroke care: compliance with guidelines and impact on inhospital stroke response. Journal of Stroke and Cerebrovascular Diseases. 2014; 23: 2773-2779.

[23] Simonsen SA, Andresen M, Michelsen L, Viereck S, Lippert FK, Iversen HK. Evaluation of pre-hospital transport time of stroke patients to thrombolytic treatment. Scandinavian Journal of Trauma, Resuscitation and Emergency Medicine. 2014; 22: 65.

[24] Puolakka T, Kuisma M, Länkimäki S, Puolakka J, Hallikainen J, Rantanen $\mathrm{K}$, et al. Cutting the prehospital on-scene time of stroke thrombolysis in Helsinki: a prospective interventional study. Stroke. 2016; 47: 3038-3040.

[25] Drenck N, Viereck S, Bækgaard JS, Christensen KB, Lippert F, Folke F. Pre-hospital management of acute stroke patients eligible for thrombolysis - an evaluation of ambulance on-scene time. Scandinavian Journal of Trauma, Resuscitation and Emergency Medicine. 2019; 27 : 3.

[26] Varjoranta T, Raatiniemi L, Majamaa K, Martikainen M, Liisanantti JH. Prehospital and hospital delays for stroke patients treated with thrombolysis: a retrospective study from mixed rural-urban area in Northern Finland. Australasian Emergency Care. 2019; 22: 76-80.

[27] Price CI, Shaw L, Islam S, Javanbakht M, Watkins A, McMeekin P, et al. Effect of an enhanced paramedic acute stroke treatment assessment on thrombolysis delivery during emergency stroke care: a cluster randomized clinical trial. JAMA Neurology. 2020; 77: 840-848.

[28] Wireklint Sundström B, Herlitz J, Hansson PO, Brink P. Comparison of the university hospital and county hospitals in western Sweden to identify potential weak links in the early chain of care for acute stroke: results of an observational study. BMJ Open. 2015; 5: e008228.

[29] Park HA, Ahn KO, Shin SD, Cha WC, Ro YS. The Effect of emergency medical service use and inter-hospital transfer on prehospital delay among ischemic stroke patients: a multicenter observational study. Journal of Korean Medical Science. 2016; 31: 139-146.

[30] Oostema JA, Chassee T, Baer W, Edberg A, Reeves MJ. Brief educational intervention improves emergency medical services stroke recognition. Stroke. 2019; 50: 1193-1200.

[31] Li T, Cushman JT, Shah MN, Kelly AG, Rich DQ, Jones CMC. Prehospital time intervals and management of ischemic stroke patients. The American Journal of Emergency Medicine. 2020; S0735-6757(20)300796.

[32] Puolakka T, Väyrynen T, Erkkilä E, Kuisma M. Fire engine support and on-scene time in prehospital stroke care - a prospective observational study. Prehospital and Disaster Medicine. 2016; 31: 278-281.

[33] Lehtonen H, Lukkarinen T, Kämäräinen V, Rautava VP, Parvinen P, Palomäki A. Improving emergency department capacity efficiency. Signa Vitae. 2016; 12: 52-57.

[34] Rautava V, Palomäki E, Innamaa T, Perttu M, Lehto P, Palomäki A. Improvement in self-reported confidence in nurses' professional skills in the emergency department. Scandinavian Journal of Trauma, Resuscitation and Emergency Medicine. 2013; 21: 16

[35] Berglund A, Svensson L, Wahlgren N, von Euler M. Face Arm Speech Time Test use in the prehospital setting, better in the ambulance than in the emergency medical communication center. Cerebrovascular Diseases. 2014; 37: 212-216.

[36] Meretoja A, Kaste M. Pre- and in-hospital intersection of stroke care. Annals of the New York Academy of Sciences. 2012; 1268: 145-151.

[37] Massé J, Dupéré S, Martin É, Lévesque MC. Transformative medical education: must community-based traineeship experiences be part of the curriculum? A qualitative study. International Journal for Equity in Health. 2020; 19: 94.

[38] Pieri M, Bellomo R, Zangrillo A, Winterton D, Landoni G. Evolving from past to future: facilitating SMART research. Signa Vitae. 2016; 12: 1418.

How to cite this article: Lauri Antila, Anne Jyrkiäinen, Lasse Hakala, Seppo Uusinarkaus, Juhani Tavasti, Ari Palomäki. Reorganisation of stroke care in prehospital emergency medical services: results through transformative learning. Signa Vitae. 2021;17(3):151-157. doi:10.22514/sv.2021.054. 